\title{
Colonisation végétale des sédiments exondés lors de la vidange du réservoir de Puyvalador : cycle biogéochimique des principaux élé- ments nutritifs
}

\author{
J. Dagnac ${ }^{1}$ \\ Ph. Le Caro ${ }^{2}$
}

Mots-clés : vidange, retenue, colonisation végétale, biomasse, minéralomasse.

Pendant l'été 1995 (juin - septembre), après la vidange du réservoir de Puyvalador (1421 m) sur la riviére Aude (Pyrénées orientales), la colonisation végétale des sédiments provisoirement exondés a été étudiée et cartographiée.

Un faible nombre d'espèces végétales participait à cette végétalisation, dont certaines avec un fort recouvrement. Une attention particulière a été portée à la biomasse et à la composition chimique des peuplements végétaux dominants, en particulier Rorippa islandica. Les mécanismes de cette colonisation et la mobilisation des principaux éléments du cycle biogéochimique font l'objet d'une discussion.

Un problème reste en suspens : l'jimpact sur la masse d'eau de ces éléments chimiques lors de la décomposition du matériel végétal une fois immergé.

Vegetal colonization of emerged sediments during the draining of the Puyvalador reservoir : biogeochemical cycle of the principal nutrients

Keywords : Draining, reservoir, vegetal colonization, biomass, mineralomass.

In summer 1995, (June - September) after the draining of the Puyvalador reservoir (1421 m) on the river Aude (Eastern French Pyrenees), colonization of the exposed sediment by vegetation was studied and cartographied. The colonization consisted of a small number of species with a large covering rate. Special attention was given to the biomass and the chemical composition of the dominant vegetation, namely Rorippa islandica. Two approaches were adopted : the mechanisms of colonization and the mobilization of the main nutrients of the biogeochemical cycle.

One problem remains uncleared : that is the impact of the chemical elements on the water column during the decay of the once-submerged vegetation.

\section{Introduction}

Comme tous les réservoirs français dont la digue a plus de 20 mètres de haut, le réservoir de Puyvalador, situé sur la rivière Aude (Pyrénées orientales), est soumis règlementairement à une visite décennale. La dernière vidange totale de ce réservoir datait de 1973 et le gestionnaire a donc été conduit à en programmer une nouvelle pendant l'été 1995. Avant et pendant les travaux, un suivi de la qualité de l'eau a été réalisé à l'aval

1. Centre d'Ecologie des Systèmes Aquatiques Continentaux, UMR C 5576, Université Paul Sabatier, 118 route de Narbonne, F 31062 Toulouse Cedex 04, France.

2. GEODE, UMR 5602, Université Toulouse Le Mirail, 5 allées Antonio Machądo, F - 31042 Toulouse Cedex 04, France. et des études ont été menées pour appréhender les incidences de la vidange sur le régime hydraulique de la rivière et le transport de sédiments. Pendant la période de 2 mois d'assec (29 juin - 3 septembre), les sédiments exondés du fond de la cuvette ont été rapidement colonisés par la végétation; cette «végétalisation» a l'avantage de fixer les sédiments tout en diminuant les possibilités de transport en cas d'orage.

Cette recolonisation végétale a été étudiée pour mieux connaître les processus, mais aussi pour en estimer l'importance et l'impact sur le cycle biogéochimique de certains éléments essentiels (Kormondy 1976, Short 1987, Labroue et al. 1994). Après la remise en eau, le matériel végétal par décomposition partielle ou totale restitue au milieu, à plus ou moins long terme, une certaine quantité d'éléments minéraux et organiques (Quennerstedt 1958, Godshalk \& Klug 1976, Suberkropp et al. 1976, Melillo et al. 1983, Pat- 
tee et al. 1985, Leuven \& Wolfs 1988, Chauvet 1989, Wetzel 1990). Cette restitution peut-elle avoir une incidence sur l'évolution future de l'écosystème vers un nouvel équilibre après la remise en eau? Quelle est l'importance de ce phénomène de redistribution par rapport aux phénomènes mieux connus de bioturbation et de remise en suspension de sédiments?

Cette première démarche a pour but d'établir un schéma méthodologique, applicable dans le futur à l'étude d'autres cas de vidange de barrage.

\section{Site, matériel et méthodes}

\section{1. Caractéristiques du site}

Des études antérieures sur le barrage de Puyvalador réunissent des données géographiques, géologiques et physico-chimiques (Dupias \& Izard 1973, Rey et al. 1973, Fabre 1988 a et b, Chassagne 1994). Nous ne transcrirons ici que les quelques valeurs importantes dans notre approche.

Le climat est de type montagnard et assez rude (Gaussen 1972), ce plan d'eau étant situé à $1421 \mathrm{~m}$ d'altitude; le pourcentage mensuel de nombre de jours de gel et de dégel est très élevé en février, mars, avril (> $60 \%$ ), encore élevé en mai (voisin de $30 \%$ ); $4,6 \%$ de jours de gel sont encore possibles en juin. La date de reprise de la végétation se trouve ainsi repoussée vers la fin juin pour les espèces les plus sensibles au froid. Les pluies sont souvent printanières (mai et juin) et, si l'on y ajoute la fonte des neiges, les sols sont généralement chargés d'eau à cette période. Ces conditions sont très sélectives pour les espèces végétales. Les vents, souvent forts, soufflent essentiellement du secteur nord/nord-ouest ( $200 \mathrm{j} / \mathrm{an}$ ) et ont un effet de transport aérien et d'accumulation des graines, mais ils peuvent avoir aussi le même effet par flottation, lorsque le barrage est en eau.

La superficie de 102 ha à la cote maximale 1421,50 NGF est relativement modeste. L'axe principal de la retenue est sud-nord; les pentes des rives sont faibles dans la partie méridionale et plus fortes dans la partie nord, surtout dans le talweg du Galbe et la rive septentrionale sous le hameau d'Odeillo. Il en résulte que les hauteurs d'eau maximales $(25 \mathrm{~m}$. si on néglige la fosse au pied du barrage près des vannes) ne concernent que la partie nord, secteur de rencontre des eaux du Galbe et de l'Aude. Le marnage étant fort, une partie importante de la cuvette, en particulier un large rivage ouest, se trouve fréquemment hors d'eau.

Les roches constituant la majorité du bassin versant $\left(134 \mathrm{~km}^{2}\right)$ sont à dominante siliceuse ou silico-alumineuse et les différents matériaux d'altération de ces roches, glaciaires ou post-glaciaires (Viers 1968), qui tapissent les versants sont à l'origine de sols de type brun acide à évolution podzolique plus ou moins poussée suivant la situation et le type de couverture végétale. Quelques affleurements de calcaire métamorphique du primaire (Fontrabiouse. danss la vallée du Galbe) sont la seule source potentielle de carbonate de calcium $\left(\mathrm{CO}_{3} \mathrm{Ca}\right)$.

Les sédiments et les colluvions qui tapissent les versants ou le fond de la cuvette de Puyvalador sont pauvres en minéraux alcalino - terreux $(\mathrm{Ca}, \mathrm{Mg})$ mais riches en aluminium et fer. Ces sédiments ont fait l'objet d'études antérieures (Fabre 1988 a, Chassagne 1994), une cartographie sommaire étant proposée par Fabre.

\section{2. Matériel et méthodes}

Les sédiments provenant en partie de l'accumulation des matières en suspension dans la colonne d'eau, ces dernières ont été caractérisées d'un point de vue chimique. Pour cela, six pièges ont été installés le 4 avril 1995 - 4 mois avant la vidange - à 2 mètres au-dessus du fond alors que le plan d'eau se trouvait à la cote $1410 \mathrm{~m}$. Ces pièges étaient des tubes en PVC de $9 \mathrm{~cm}$ de diamètre intérieur et $50 \mathrm{~cm}$ de longueur (soit une surface de réception de $63,5 \mathrm{~cm}^{2}$ ) stabilisés par un lest et des flotteurs. Ils avaient été utilisés dans des lacs de gravière (Helmer de Almeira 1994). Ces pièges ont été rélevés une fois par mois et après 15 jours en juin. Les dépôts récupérés ont été séchés en étuve à $105^{\circ}$ jusqu'à poids constant pour la détermination du poids sec et pour les analyses des éléments totaux. Après le 15 juin, le plan d'eau se trouvant à la cote $1400 \mathrm{~m}$, nous avons interrompu le suivi.

Les analyses chimiques ont consisté à extraire les éléments minéraux totaux par un passage au four à $550^{\circ}$ après pré-digestion à l'acide nitrique; après repri-. se des résidus à l'acide chlorhydrique $(\mathrm{HCl})$, les différents éléments ont été dosés, soit par colorimétrie à flux continu (P), soit par absorption atomique $(\mathrm{Ca}, \mathrm{Mg}$, $\mathrm{K}, \mathrm{Fe}, \mathrm{Mn}$ ). Le carbone (C) et l'azote $(\mathrm{N})$ ont été analysés par voie chimique. La détermination des concentrations en carbone organique et azote total est réalisée, sur la même prise d'essai, après attaque à chaud par un mélange d'acide sulfurique et de bichromate de potassium. Les dosages par titration d'un aliquote de la solution d'attaque sont faits par le sel de Mohr pour le carbone et l'acide sulfurique, après distillation en présence de soude, pour l'azote.

Sur le matériel végétal, les mêrnes méthodes d'extraction et de dosage ont été utilisées. 
A chacune des visites du site, la zonation de la végétation s'imposait avec une telle évidence qu'une cartographie sommaire paraissait le meilleur moyen de parvenir à une stratification de l'échantillonnage des biomasses végétales. Deux cartes de végétation, à deux stades différents de la colonisation, ont été établies; leur réalisation a été rendue possible grâce à deux photographies aériennes prêtées par Electricité de France (EDF).

La première photographie couleur verticale effectuée par IGN en début d'assec a été ramenée à l'échelle de travail par agrandissement et a permis de reporter différentes limites utiles pour les deux stades. La deuxième phọtographie couleur, oblique mais panoramique, prise d'hélicoptère vers la mi-août nous a grandement facilité l'établissement de la deuxième carte de végétation. Tout cela a été complété par de nombreuses prises photographiques au sol lors des relevés de végétation réalisés pendant la période estivale. Les deux cartes ébauchées manuellement ont ensuite été numérisées et mises en forme définitivement à l'aide du logiciel Photoshop.

Les espaces occupés par ces différents ensembles ont pu être déterminés après numérisation des deux cartes à l'aide d'un scanner. Les deux fichiers images ont été ensuite traités à l'aide du logiciel PC-IMAGE pour le calcul des différentes superficies d'occupation du sol. Un parcours des zones émergées, commandé par la zonation perçue, a permis de dresser une liste globale de chaque faciès rencontré. Une attention plus soutenue a été portée aux végétations de forte biomasse, les plus susceptibles, a priori, de contribuer significativement au bilan biogéochimique, objectif de l'étude. Le temps disponible ne permettait pas des relevés complets de végétation.

Dans les zones choisies pour l'échantillonnage des biomasses végétales des espèces dominantes, le nombre de pieds au mètre carré a été obtenu par comptage dans trois carrés de $100 \mathrm{~m}^{2}$ dans les zones ouvertes et $1 \mathrm{~m}^{2}$ dans les zones de végétation très dense; 3 à 5 individus jugés représentatifs (sans validation statistique) ont été prélevés pour pesées et analyses.

\section{Résultats}

\section{1 Sédiments et matières en suspension}

Les données de la bibliographie ont été complétées par l'analyse de 10 prélèvements personnels.

Les dépôts réellement représentatifs de sédiments fins limoneux, d'une épaisseur guère supérieure à 25 $\mathrm{cm}$, sont surtout localisés dans la cuvette principale qui correspond à la courbe de niveau $1406 \mathrm{~m}$. Vers l'amont du plan d'eau, seule une mince couche de dépôts lacustres ( 2 à $10 \mathrm{~cm}$ d'épaisseur) repose sur les alluvions de l'ancienne terrasse de l'Aude actuellement submergée toute l'année, sauf dans l'extrême partie amont. Pour le reste, c'est-à-dire la majeure partie du talus soumis chaque année à un fort marnage, souvent supérieur à 12 mètres et pouvant atteindre 17 mètres en avril et mai, les dépôts sont hétérogènes et vont du pôle de dépôts très grossiers (galets, graviers, sables) à un pôle de dépôts plus fins, sablo-limoneux. Il existe en rive gauche, généralement en bas du talus, d'anciennes tourbières de pente d'âge post-glaciaire. Des affleurements de tourbe identiques se rencontrent plus rarement en haut du talus rive droite en contrebas des colluvions du versant d'Odeillo. Ce versant rive droite affiche une pente forte $\left(\right.$ de $10^{\circ}$ à $25^{\circ}$ ) formée en grande majorité de dépôts sableux avec moins de $0,7 \%$ de fraction $<50 \mu$.

Notons enfin la particularité des dépôts du fond du talweg du Galbe, qui s'accumulent souvent sur plus d'un mètre d'épaisseur ; ces dépôts, typiques des cônes de déjections torrentiels, sont très nettement stratifiés avec une alternance de niveaux sableux ou limoneux de 10 à $30 \mathrm{~cm}$ avec des niveaux (trois repérés dans notre coupe) de 2 à $6 \mathrm{~cm}$ de débris végétaux grossiers de feuilles et brindilles incomplètement décomposées. Ces niveaux correspondent à des périodes torrentielles paroxismiques pouvant être liées à des phénomènes d'avalanches en amont du bassin versant et difficiles à dater précisément.

Les sédiments fins de la cuvette centrale et de la terrasse alluviale de l'Aude proviennent de l'accumulation des matières en suspension de la colonne d'eau, lors des périodes de calme favorables. Les remises en suspension doivent être fréquentes, surtout sur les talus au moment des épisodes de marnage par fort vent et donc, forte agitation de l'eau par les vagues.

Les six pièges répartis dans la cuvette principale ont permis de recueillir en moyenne par piège :

du 1 - 30 avril : $2,10 \mathrm{~g}$ de matière sèche.

du 1 - 30 mai : 3,19 $\mathrm{g}$ de matière sèche.

du 1 - 15 juin : 2,22 g de matière sèche.

A partir de ces valeurs, une première estimation du bilan des dépôts donne un rythme d'environ $50 \mathrm{t}^{-1} \mathrm{ha}^{-1}$ $1 \mathrm{an}^{-1}$ de matières en suspension pour le secteur central de la cuvette. Cependant, cette méthode de détermination des bilans des dépôts sédimentaires en milieu lacustre reste encore imprécise (Butcher \& al. 1993, Dagnac 1994).

Pour les analyses chimiques, les échantillons des 6 pièges ont été mélangés. Les résultats d'analyses des 
teneurs en éléments minéraux essentiels dans différentṣ compartiments de l'écosystème étudié sont résumés dans le tableau 5. Dans l'ensemble, les valeurs pour les MES sont voisines de celles que l'on peut rencontrer dans la littérature. Il en va de même pour les valeurs obtenues dans l'eau concernant les cations participant à la balance ionique, essentiellement $\mathrm{Ca}^{++}$, $\mathrm{Mg}^{+}, \mathrm{K}^{+}, \mathrm{Fe}^{++}$(Meybeck 1976). Comme déjà souligné par Mackereth (1957), on a constaté l'immobilisation des éléments essentiels dans le sédiment; ceci étant net pour le potassium $(\mathrm{K})$ et le calcium $(\mathrm{Ca})$ et encore plus marqué pour le manganèse ( $\mathrm{Mn}$ ), deux fois plus abondant dans le sédiment que dans les MES et $10^{3}$ fois plus abondant dans le sédiment que dans l'eau du lac. Les valeurs moyennes obtenues pour cinq échantillons de sédiment en place sur le fond du grand talweg de l'Aude sont tout à fait comparables à celles obtenues pour les MES et les sédiments d'autres lacs de barrages - Pareloup, Bage et Pont de Salars'dans l'Aveyron)-, dans un même environnement de roches cristallines (Dagnac 1994, David 1994). Globalement, le stock minéral total laisse supposer une disponibilité importante pour la nutrition végétale.

\section{2. La colonisation végétale du bassin}

Deux stades dans la végétalisation des espaces découverts ont été cartographiés :

- le premier stade au 1er juillet 1995 correspond à la situation avant vidange totale (Fig. 1A);

- le deuxième stade au ler septembre 1995 trois jours avant la remise en eau du réservoir (Fig. 1B).

La nomenclature botanique utilisée est celle de Bolos et al. (1990). On trouvera à la fin de l'article une liste récapitulative des espèces citées (Tableau 7).

\section{2. 1. Situation au 1er juillet 1995 (Fig. 1A)}

Sur la rive gauche du lac, en pente faible, la zonation rappelle celle des anses maritimes du littoral atlantique soumises à des submersions temporaires, cycliques et de durée graduée. C'est pourquoi nous avons dénommé la ceinture tout-à-fait supérieure «zone du haut schorre en voie de boisement». Rarement submergée, mais par places toujours humide (mouillères), c'est une étroite zone enherbée en permanence, avec une végétation diversifiée, comprenant quelques espèces landicoles et beaucoup d'hygrophiles (Baudière \& Serve 1976, Terrisse \& Terrisse 1976).

On remarque surtout :

Filipendula ulmaria

Deschampsia caespitosa

Leontodon autumnalis

Genista anglica

\section{Rorippa islandica}

Briza media

Achillea ptarmica subsp. pyrenaica

Ranunculus repens

Lotus sp.

Juncus cf.conglomeratus

Galium sp. cf. pumilum

avec des individus, parfois groupés, de :

$X$ Pinus sylvestris $\mathrm{x} P$. mugo subsp. uncinata, Betula pubescens, Salix sp.pl. et tout particulièrement $S$. pentandra et Populus nigra (individus chétifs).

D'autres plantes, plus dispersées, reflètent le même spectre écologique :

Equisetum sp.(pl. ?)

Lychnis flos-cuculi

Orchis majalis

Carex sp. pl.

Prunella vulgaris

Glyceria fluitans

Trifolium sp. pl.

Chamaespartium sagittale

Armeria alliacea

Galium verum subsp. verum

Holcus mollis

La zone à Deschampsia et Leontodon occupe une superficie voisine de 6 ha. Comprise entre les courbes 1418 et $1420 \mathrm{~m}$, elle est donc concernée par la fluctuation annuelle du rivage (Quennerstedt 1958). Cette zone est souvent découverte pendant l'été (graphiques 1992 et 1994, Fig. 2); la pente y est très faible. Le substrat correspond davantage à un sol qu'à un sédiment : il est constitué d'une terre fine tapissée en surface d'une mince couche de graviers anguleux et souvent aplatis.

Ce groupement permanent est composé de plusieurs espèces végétales constituant une pelouse presque fermée dans la partie haute (peuplement dense de Deschampsia caespitosa), très largement ouverte en partie basse. Vers le haut, à l'exception notable des pins, la flore reste à peu près celle du «haut schorre»; vers le bas, la liste des plantes est très réduite. En plus des deux espèces éponymes on citera les individus dispersés de Paronychia polygonifolia et de Poa compressa : végétation de plantes vivaces durement sélectionnées par l'alternance submersion - dessèchement (Fraissé \& Maman 1993).

Une zone végétalisée, assez importante en queue de retenue, à laquelle s'ajoutent ponctuellement trois petits secteurs, bien localisés, se distingue nettement dès le ler juillet 1995 et avant même la vidange. Depuis la mi-février 95 jusqu'au début juin, le plan d'eau a été 

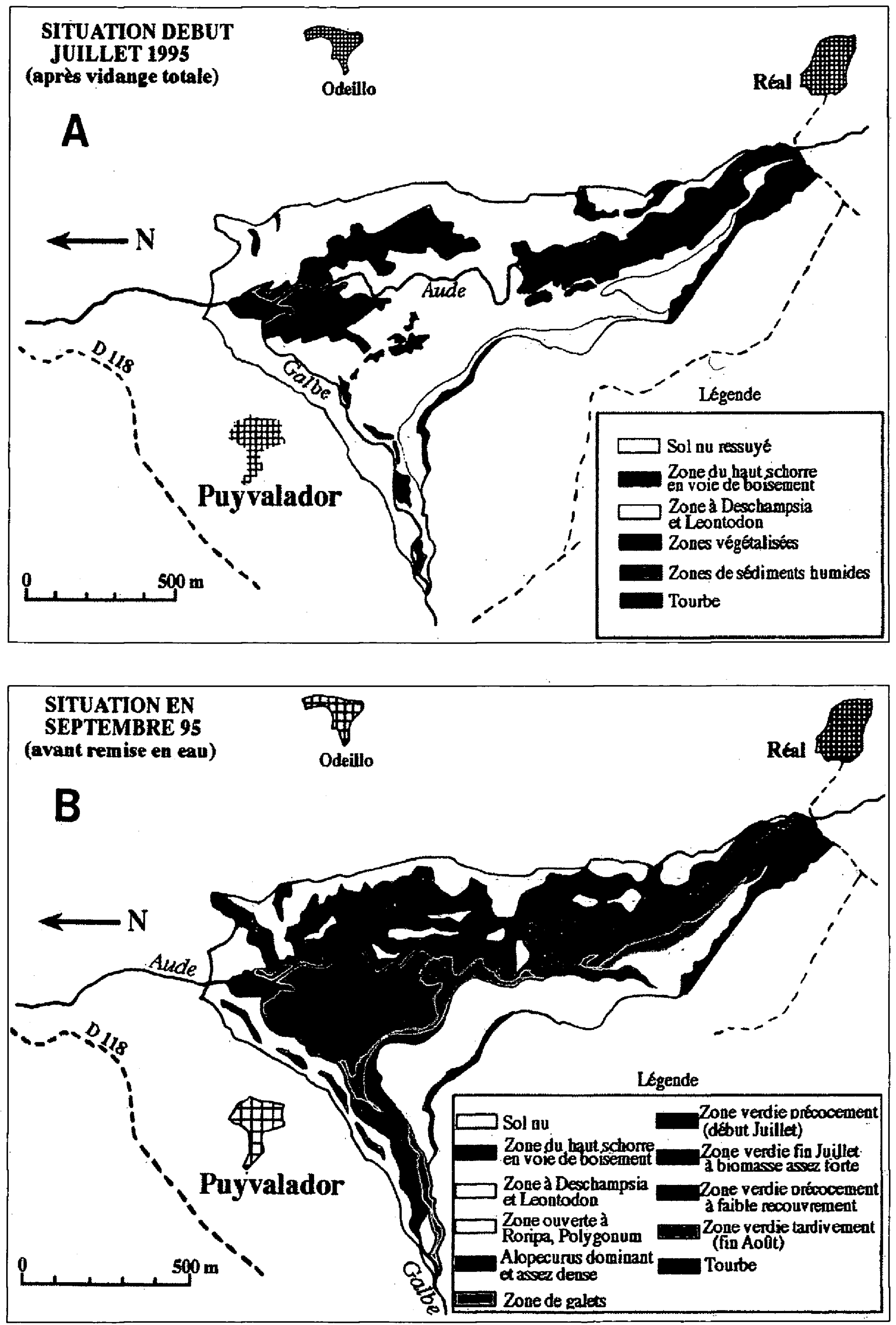

Fig. 1. Deưx stades de colonisation végétale après vidange totale du réservoir. 1A. Situation début juillet 1995. 1B. Situation en septembre 1995. Fig. 1. Two stages of vegetational colonization after draining of the reservoir. 1A. State at the beginning of July 1995. 1B. State in September 1995. 


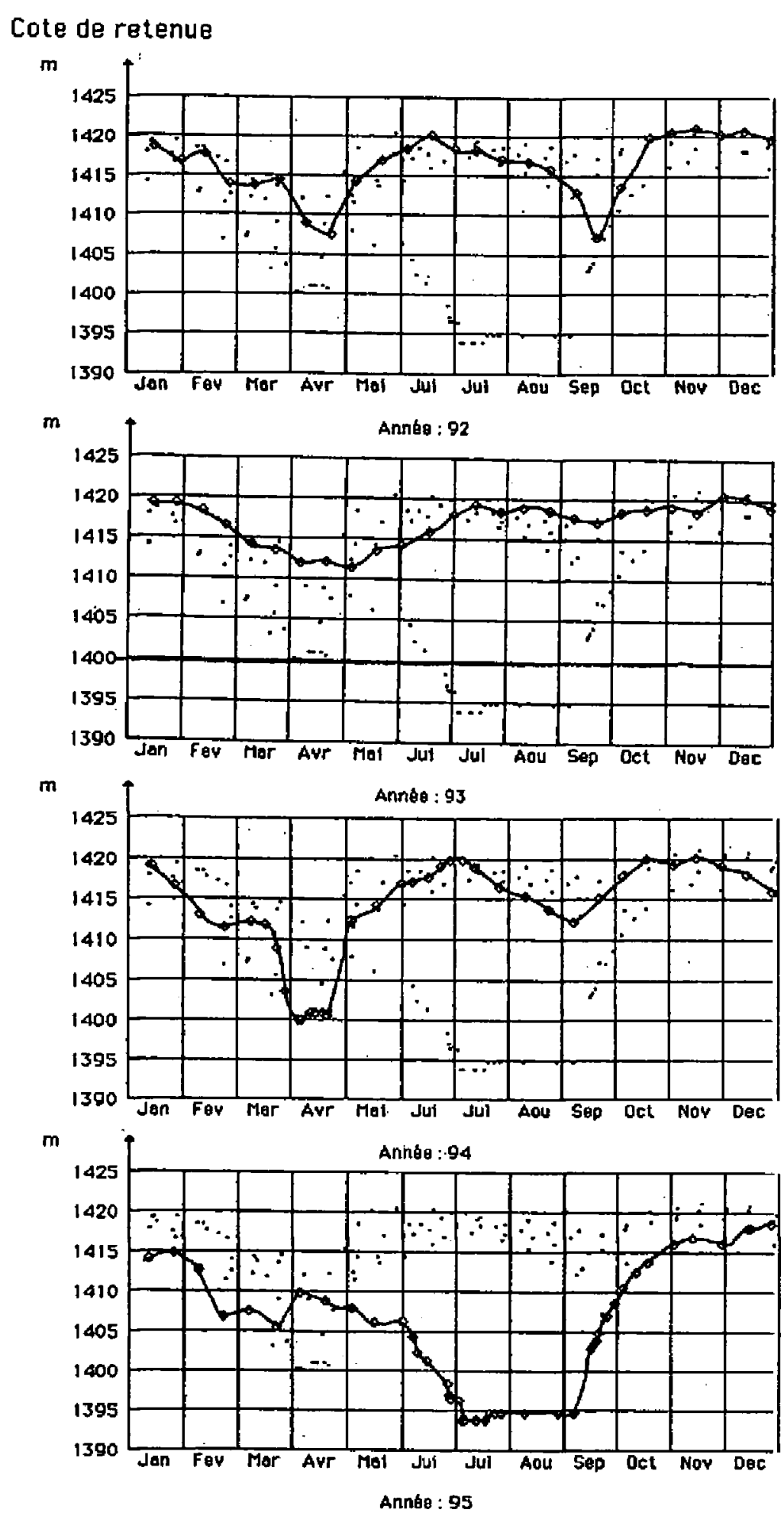

Fig. 2. Variations annuelles de la cote de la retenue de Puyvalador. Fig. 2. Annual variation of Puyvalador reservoir level.

exceptionnellement maintenu à un niveau assez bas (inférieur à la cote 1410) (Fig. 2).

Les zones propices à la végétation ont donc pu être colonisées très tôt. Notons que cette cote $1410 \mathrm{~m}$ peut être souvent atteinte en avril puisqu'elle est légèrement supérieure à la cote minimale d'exploitation de $1399,4 \mathrm{~m}$.

Il s'agit là d'un tout autre type de végétation : celui des superficies toujours en eau colonisées exception- nellement lors de cette vidange totale. Trois espèces seulement constituent dès le début l'essentiel de la biomasse : Alopecurus geniculatus subsp. fulvus, Rorippa islandica et Gnaphalium uliginosum ; à côté desquelles il faut citer, remplaçant par places Alopecurus, le Catabrosa aquatica repérable par ses longs stolons rampants aux gaines rouges. Une quasi-absence remarquable est celle du Polygonum persicaria, qui sur un support encore très humide et donc froid n'est probablement pas concurrentiel en début de saison.

Les espèces plus rares sont diversifiées, mais leur participation en terme de biomasse est négligeable. Il s'agit surtout de rudérales, de plantes de friche humide, ou d'habituées des bords des ruisseaux, par exemple :

Polygonum aviculare

Galeopsis sp.

Descurainia sophia

Matricaria maritima . subsp. inodora

Arabidopsis thaliana.

Thlaspi arvense. subsp. arvense

Capsella bursa-pastoris

Typha sp.

Poa trivialis

Ranunculus repens

Epilobium cf. hirsutum

Juncus sp.

Stellaria alsine

Veronica beccabunga

Ranunculus (subgen. Batrachium) sp.

Deux plantules occasionnelles surprennent dans un tel environnement, encore marqué par l'hygrophilie : Armeria alliacea et Artemisia absinthium.

Pour le reste du bassin, le ressuyage des sols et des sédiments allait en s'accentuant et pouvait permettre l'installation d'espèces adaptées aux modalités et à l'intensité de l'assèchement (Langlade 1996).

Les zones trop en pente, comme le versant sous le hameau d'Odeillo ou celui sous le village de Puyvalador, avec un substrat trop grossier (galets, graviers, sables grossiers), trop percolant ou trop pauvre en fractions fines, étaient plus difficilement colonisées par quelques espèces à faible recouvrement.

\section{2. 2. Situation au ler septembre 1995 (Fig. 1B)}

La carte montre qu'en dehors d'une surface de sol nu, généralement à forte pente $(>20 \%)$ et constituée de matériaux grossiers, d'une zone de gros galets soulignant le bas du talus occidental à faible pente, et d'affleurements de tourbe postglaciaire, on peut différencier huit groupements végétaux de composition ou d'évolution différentes. 
Aux trois ensembles végétalisés représentés sur la première carte (Fig. 1A), s'ajoutent cinq nouvelles zones :

1) une zone ouverte à Rorippa et Polygonum qui forme une plage à végétation dispersée où les plantes, très isolées en début de saison, laissent encore en septembre $75 \%$ de sol nu.

Les deux plantes dominantes sont deux annuelles des lieux humides ouverts : Rorippa islandica et Polygonum persicaria accompagnées par Alopecurus geniculatus (vieux pieds ayant survécu aux cycles précédents d'émersion - submersion, et jeunes individus provenant d'une germination 1995.)

La flore accompagnatrice, négligeable en volume, est d'une grande diversité spécifique et biologique ; en plus des hygrophiles déjà remarquées sur les berges du lac, apparaissent de très nombreuses plantes des sols nus : rudérales, adventices ou mêmes messicoles (Bourraqui-Sarre 1997), accompagnées de diverses espèces venues de tel ou tel biotope spécialisé du bassin versant. On citera notamment des semis de : Betula pubescens et de Salix sp. ainsi que :

Deschampsia caespitosa (en haut de la zone)

Juncus sp. pl.

Mentha longifolia

Achillea pyrenaica

Polygonum aviculare

Spergularia rubra subsp. rubra

Sagina apetala subsp. erecta

Poa annua

Conyza cf. canadensis

Rumex sp.(subgen. Rumex)

Anthyllis gr. vulneraria

Capsella bursa-pastoris

Lamium amplexicaule

Spergula sp.

Scleranthus sp.

Urtica dioica

Sonchus sp.(annuel)

Solanum lycopersicum

Senecio vulgaris

Poa pratensis

Artemisia absinthium

2) une zone à Alopecurus dominant et assez dense contrastant avec la précédente par la densité du couvert (80 à $100 \%$ ), mais non par la liste floristique. Alopecurus est dominant, avec beaucoup de pieds préexistants à la vidange, et très vraisemblablement ayant survécu aux immersions et assecs successifs.

Les plantes «accidentelles» sont les mêmes que celles de la zone 1 avec en plus : Juncus bufonius, $M a$ - $^{-}$ tricaria maritima subsp. inodora, Artemisia vulgaris.
3) une zone verdie fin juillet à biomasse assez forte comporte une végétation très analogue à celle de la zone cartographiée comme végétalisée en début juillet : il s'agit du même biotope et du même verdissement, mais décalé dans le temps (ou mieux, dans la saison) par la date d'émersion.

Alopecurus prédomine, Rorippa, Polygonum persicaria et Polygonum aviculare occupent le reste de l'espace, le plus souvent en peuplement mélangé.

Les plantes accompagnatrices, en individus dispersés au hasard des germinations, sont le reflet de la végétation environnante : notamment Tanacetum vulga$r e$, plante très abondante en Cerdagne et Capcir.

4) une zone verdie précocement dans le cours de l'été, mais à faible recouvrement végétal, concerne la périphérie de la cuvette centrale du lac.

La plante dominante est soit Rorippa soit Alopecurus. Polygonum aviculare et plusieurs Juncus sont fréquents.

5) une zone verdie tardivement (fin août) correspond à la très basse plaine du fond de la cuvette du lac et sensiblement aux sédiments qui, émergés, étaient encore gorgés d'eau début juillet.

Début septembre, apparait une dense prairie - ou plutôt un champ - de Rorippa encore essentiellement à l'état végétatif, ou en début de floraison. La densité du peuplement est impressionnante, analogue à celle d'un champ de colza en début de montaison au printemps. Sur 3 placettes de $1 \mathrm{~m}^{2}$, nous avons dénombré en moyenne 40 pieds $/ \mathrm{m}^{2}$.

Le tableau 1 récapitule des pourcentages d'occupation du sol en début et en fin d'exondation de la cuvette.

\section{2 .3. Le stock de graines}

L'extraordinaire verdissement constaté début juillet pose un problème important mais difficile à appréhender à savoir le mécanisme d'ensemencement naturel des surfaces exondées. On pouvait penser à un transfert éolien ou un transport par flottation par la masse d'eau en régression. C'est cette dernière hypothèse que nous avons retenue.

Ce problème a d'ailleurs intrigué Terrisse (1996) qui apporte à nos listes floristiques des compléments importants.

Notons que dès la mi-juillet 94 , toutes les surfaces au dessus de $1415 \mathrm{~m}$. sont restées exondées tout l'été ; dans cette zone, les végétaux colonisateurs ont pu accomplir leur cycle normal jusqu'à la fructification et fournir ainsi un volume important de graines pour la saison suivante. Les graines tombées au sol ont été re- 
Tableau 1. Pourcentage d'occupation du sol en juillet et septembre 1995 des différents milieux cartographiés du réservoir de Puyvalador pour une surface totale exondée de 102 ha de bassin.

Table 1. Importance of various land-survey classes in the Puyvalador reservoir in Julyand Septembre 1995 as a part of a total drained surface $102 \times 10^{-4} \mathrm{~m}^{2}$.

\begin{tabular}{|c|c|c|c|}
\hline Début Juillet & $\%$ & Septembre & $\%$ \\
\hline Sols ressuyés & 70,1 & Solnu & 21,4 \\
\hline Zone du haut schorre en voie de boisement & 3,1 & Zone de haut schorre en voie de boisement & 3,1 \\
\hline Zone à Deschampsia et Leontodon & 5,0 & Zone à Deschampsia et Leontodon & 8,5 \\
\hline Zones végétalisées & 10,2 & Zone ouverte à Rorippa et Polygonum & 8,9 \\
\hline \multirow[t]{6}{*}{ Zones de sédiments humides } & 10,2 & Zone à Alopecurus dominant et assez dense & 3,2 \\
\hline & & Zone de galets & 7,4 \\
\hline & & Zone verdie précocément (début juillet) & 10,4 \\
\hline & & Zone verdie fin juillet a biomasse assez forte & 14,9 \\
\hline & & Zone verdie prócocément faible recouvrement & 9,5 \\
\hline & & Zone verdie tardivement (fin août) & 11,3 \\
\hline Tourbe & 0,6 & Tourbe & 0,6 \\
\hline Eau & 0,8 & Eau restante & 0,8 \\
\hline
\end{tabular}

prises par l'eau qui est remontée à l'automne et qui les a disséminées par flottation dans tout le bassin. Dès la fin des gelées printanières ces graines ont germé au fur et à mesure que les surfaces de sédiments se découvraient et que les conditions environnementales le permettaient. Cependant localement, une dissémination par le vent n'est pas exclue. Dans des sédiments du même réservoir, prélevés en immersion à la benne Eckman, lors d'une étude préliminaire en avril 94, dans les refus de tamisage granulométrique supérieurs à $500 \mu \mathrm{m}$, se trouvait un nombre important de graines d'une espèce non déterminée. La germination, lors de l'assec, de graines provenant d'une alimentation régulière du sédiment, dans les conditions habituelles du fonctionnement du plan d'eau, ne peut donc être exclue. Dès la première semaine de juillet, les sédiments de fond, amenés à l'air, se sont ressuyés progressivement tandis que germaient parallèlement les graines qu'ils renfermaient. A la fin du mois d'août 95 , la couverture végétale était déjà importante (Fig. 1B).

\section{3. Estimation des biomasses végétales et des mi- néralomasses}

\section{3. 1. Les biomasses végétales}

Seule la biomasse aérienne est considérée, le système racinaire des végétaux analysés n'est pas été pris en compte.
Le tableau 2 donne les poids secs de plants de $R o$ rippa au stạde de maturité recueillis dans trois peuplements (I, II, III) pendant la deuxième quinzaine d'août.

Au séchage, un pied de Rorippa frais peut perdre plus de $85 \%$ de son poids. Pour cette même espèce, les feuilles représentent $37,5 \%$ du poids sec de la plante, les tiges $62,5 \%$, les inflorescences une part négligeable.

Les variations de biomasse aérienne pour différentes espèces peuvent être importantes (Tableau 3). La biomasse moyenne la plus faible correspond au petit Polygonum aviculare plante grêle et rampante à très petites feuilles presque rondes et rares ; par contre, celle de Polygonum persicaria, espèce de grande taille à grandes feuilles lancéolées avec une tige d'un centimètre de diamètre est huit fois plus forte.

La graminée Alopecurus geniculatus montre de faibles écarts de biomasse entre les pieds; ceci étant dû à la structure généralement plus homogène de ce genre.

\section{3. 2. Les éléments minéraux chez Rorippa islandica}

Rorippa islandica domine largement dans les différents groupements pendant l'été 95 ; elle peut présenter jusqu'à $100 \%$ de recouvrement.

C'est à partir de cette espèce que nous avons essayé d'estimer l'importance de la restitution d'éléments minéraux essentiels au milieu après remise en eau. 
Tableau 2. Biomasses des individus de Rorippa prélevés dans les trois peuplements échantillonnés..

Table 2. Dry weigh biomass of Rorripa specimen in three selected stands.

\begin{tabular}{|c|c|c|c|}
\hline \multicolumn{2}{|c|}{ Peuplement I } & \multicolumn{2}{|c|}{ Peuplement II } \\
\hline Individu & Poids sec/g de MS & Individu & Poids sec/g de MS \\
\hline A & 10,27 & E & 7,66 \\
\hline $\mathrm{B}$ & 15,83 & $\mathrm{~F}$ & 6,76 \\
\hline $\mathrm{C}$ & 7,60 & G & 11,62 \\
\hline D & 8,22 & Moy. & 8,68 \\
\hline Moy. & 10,48 & \multicolumn{2}{|c|}{ (pieds peu vigoureux) } \\
\hline \multicolumn{4}{|c|}{ Peuplement III } \\
\hline Individu & Poids sec/g de MS & Perte & eau des pieds frais \\
\hline $\mathrm{H}$ & 16,55 & & 82,6 \\
\hline 1 & 12,20 & & 84,8 \\
\hline $\mathbf{J}$ & 5,86 & & 86,2 \\
\hline $\mathrm{K}$ & 8,57 & & 87,8 \\
\hline Moy. & 10,80 & & 85,35 \\
\hline
\end{tabular}

Tableau 3. Biomasse aérienne de trois espèces bien représentées dans la zone d'étude.

Table 3. Aerial biomass of three dominating species.

\begin{tabular}{cccc}
\hline échantillon & $\begin{array}{c}\text { Polygonum aviculare } \\
\text { en g/MS }\end{array}$ & $\begin{array}{c}\text { Polygonum persicaria } \\
\text { en g/MS }\end{array}$ & $\begin{array}{c}\text { Alopecurus en } \\
\text { g/MS }\end{array}$ \\
\hline 1 & 12,97 & 44,70 & 9,17 \\
2 & 10,24 & 61,95 & 7,65 \\
3 & 9,41 & 50,62 & 8,82 \\
4 & 5,70 & - & - \\
Moy. & 6,34 & 52,42 & 8,55 \\
\hline
\end{tabular}

Quatre éléments majeurs ( $\mathrm{P}, \mathrm{Ca}, \mathrm{Mg}, \mathrm{K}$ ) et deux mineurs ( $\mathrm{Fe}, \mathrm{Mn})$. ont été retenus en considérant le rôle prioritaire qu'ils jouent à côté de l'azote $(\mathrm{N})$ et du carbone (C) dans le cycle biogéochimique et les échanges sédiments - eau (Mas 1959, Smith 1962, , Gerloff \& Fishbeck 1969, Hutchinson 1975, Kem-Hansen \& Dawson 1978, Allenby 1981, Petitjean 1981, Gerloff \& Krombholz 1996).

Le tableau 4 résume les principaux résultats obtenus pour les feuilles et les tiges de 4 pieds de Rorippa dont les biomasses ont été déterminées préalablement (échantillons H, I, J, K du tableau 2).
Pour tous les éléments analysés, les feuilles présentent toujours des concentrations plus fortes que les tiges. Le potassium est l'élément prépondérant; il est presque aussi concentré dans les tiges que dans les feuilles alors que les teneurs varient du simple au double pour les autres éléments. On remarque les très faibles teneurs en fer et manganèse dans les tiges et des variations importantes pour le calcium. Le tableau 5 permet de comparer les teneurs en éléments minéraux pour différents compartiments de l'écosystème. Une partie importante du phosphore des MES provient de la matière organique (plancton) et l'on voit que les vé- 
Tableau 4. Teneurs en éléments minéraux des feuilles et des tiges de 4 échantillons $(\mathrm{H}, \mathrm{I}$, $\mathrm{J}, \mathrm{K}$ du tableau 2) de Rorippa islandica en \% de matière sèche.

Table 4. Leaves and stems mineral elements contents of Rorripa islandica (the sames specimens, H, I, J, K en table 2 - \% DM unit).

\begin{tabular}{|c|c|c|c|c|c|c|}
\hline \multicolumn{7}{|c|}{ Echantillon de feuilles } \\
\hline & $\mathbf{P}$ & $\mathbf{C a}$ & $\mathbf{M g}$ & $\mathbf{K}$ & $\mathbf{F e}$ & Mn \\
\hline 1 & 0,34 & 1,21 & 0,39 & $\overline{2,30}$ & 0,35 & 0,012 \\
\hline 2 & 0,45 & 1,03 & 0,34 & 2,70 & 0,28 & 0,011 \\
\hline 3 & 0,46 & 1,05 & 0,35 & 3,10 & 0,40 & 0,013 \\
\hline 4 & 0,44 & 0,99 & 0,46 & 2,95 & 0,17 & 0,008 \\
\hline Moy. & 0,42 & 1,07 & 0,39 & 2,76 & 0,30 & 0,011 \\
\hline \multicolumn{7}{|c|}{ Echantillon de tiges } \\
\hline & $\mathbf{P}$ & $\mathbf{C a}$ & $\mathbf{M g}$ & $\mathbf{K}$ & $\mathbf{F e}$ & $\mathbf{M n}$ \\
\hline 1 & 0,26 & $\overline{0,69}$ & 0,18 & 2,10 & 0,07 & 0,004 \\
\hline 2 & 0,27 & 0,29 & 0,13 & 2,40 & 0,09 & 0,004 \\
\hline 3 & 0,32 & 0,34 & 0,15 & 2,65 & 0,08 & 0,005 \\
\hline 4 & 0,23 & 0,41 & 0,19 & 2,45 & 0,08 & 0,004 \\
\hline Moy. & 0,27 & 0,43 & 0,16 & 2,40 & 0,08 & 0,004 \\
\hline
\end{tabular}

Tableau 5. Teneurs comparées des principaux éléments minéraux dans divers compartiments de l'écosystème (valeurs moyennes pour les matières en suspension (MS) obtenues pour le réservoir de Puyvalador).

Table 5. Major chemical elements in some sections of Puyvalador reservoir biogeocoenosis.

\begin{tabular}{|c|c|c|c|c|c|}
\hline \multicolumn{2}{|c|}{ Eléments } & Teneur dans & \multirow{2}{*}{$\begin{array}{l}\text { Matières en suspension } \\
\text { (MS) }\end{array}$} & \multirow{2}{*}{$\begin{array}{l}\text { Sédiments fins de la } \\
\text { cuvette centrale (MS) }\end{array}$} & Végétaux (Rorippa \\
\hline & & l'eau en $\mathrm{mg} / \mathrm{t}$ & & & plante entière) (MS \\
\hline $\mathbf{P}$ & $\% o$ & 0,30 & 1,30 & 0,7 & 3,30 \\
\hline $\mathrm{K}$ & $\%$ & 0,80 & 0,56 & 2,25 & 2,50 \\
\hline $\mathrm{Ca}$ & $\%$ & 17,2 & 0,17 & 0,30 & 0,70 \\
\hline $\mathrm{Mg}$ & $\%$ & 2,3 & 0,47 & 1,60 & 0,25 \\
\hline $\mathrm{Fe}$ & $\%$ & $0,05-0,50$ & 4,13 & 4,39 & 1,70 \\
\hline Mn & $\%$ & $0,001-0,12$ & 0,10 & 0,22 & 0,07 \\
\hline C & $\%$ & - & $.6,50$ & 4,53 & 35,0 \\
\hline & $\%$ & - & 0,60 & 0,41 & 3,6 \\
\hline
\end{tabular}

gétaux supérieurs, comme Rorippa, le concentrent fortement, alors que dans le sédiment il est 4 fois moins important. Le calcium qui joue un rôle physiologique majeur chez la plante est également concentré ; il est deux fois moins abondant dans le sédiment. Par contre, le magnésium, constituant essentiel de la chlorophylle, qui représente en moyenne $0,40 \%$ de la matière sèche chez les végétaux, présente chez Rorippa une valeur plutôt faible alors que son taux dans le sédiment est conforme à ce que l'on trouve habituellement. Suivant l'état de la plante et les espèces, les différents éléments peuvent varier assez fortement.

\section{3. 3. Expérience de décomposition en laboratoire}

En laboratoire, pendant cinq jours, une tentative de suivi du relargage d'éléments minéraux pour un pied de Rorippa frais ( $75 \mathrm{~g}$ ) maintenu dans un cristallisoir de deux litres d'eau déminéralisée ( $\mathrm{pH} 5,70$ ) sous bullage d'air a été effectuée. 
Les résultats de cette expérience résumés dans le tableau 6 montrent la chronologie de la mise en solution des différents éléments. Le pH et la conductivité croissent régulièrement au fur et à mesure du relargage des différents ions. Après vingt minutes seulement la teneur du milieu liquide en potassium a plus que décuplé et la concentration en ion $\mathrm{SO}_{4}$ est 7,5 fois supérieure à la valeur initiale.

Les teneurs en $\mathrm{NH}_{4}, \mathrm{Ca}$ et $\mathrm{Mg}$ augmentent régulièrement puis fortement en fin d'expérience.

Les orthophosphates $\left(\mathrm{HPO}_{4}\right)$ ne présentent des concentrations notables qu'en fin d'expérience (vers le 4ème jour), cependant la valeur maximale atteinte le cinquième jour est forte $(1220 \mu \mathrm{g} / \mathrm{l})$ si on la compare aux valeurs généralement rencontrées dans les eaux naturelles. L'expérience a été arrêtée prématurément devant l'impossibilité de contrôler précisément un certain nombre de paramètres importants comme la température, l'oxygène, l'activité microbienne, etc...

Nous avons pu noter que, dès le début, la plante immergée restitue rapidement le potassium, qui pourrait s'avérer un traceur intéressant à suivre lors des premiers jours de la mise en eau du réservoir.

Ces résultats préliminaires montrent qu'un tel type d'approche, mieux contrôlé et répété, pourrait contribuer à une meilleure connaissance des processus de recyclage des principaux éléments dans le milieu aquatique, lors de la décomposition des végétaux, qui suit la remise en eau d'une retenue.

\section{Discussion et conclusion}

La décomposition du matériel végétal peut s'étaler sur une période plus ou moins longue en fonction des conditions du milieu et de l'activité des microorganismes. Au bout de combien de temps tout le matériel végétal aura-t-il été minéralisé ? C'est une question à laquelle, pour le moment, nous ne pouvons répondre.

Pour une première tentative d'évaluation des quantités globales de matière potentiellement relargable dans le milieu, on peut prendre l'espèce végétale la plus fréquente.

Si un pied bien venu de Rorippa représente $10,5 \mathrm{~g}$ de matière sèche (MS), un groupement dense de Rorippa de 40 pieds au $\mathrm{m}^{2}$ correspondrait à $420 \mathrm{~g}$ de $\mathrm{MS} / \mathrm{m}^{2}$.

Pour la seule zone verdie précocement début juillet qui représente $10,4 \%$ de la surface totale soit environ 10 hectares, on aurait 42 tonnes de matière sèche végétale. Pour un rapport feuilles/tiges de $2 / 3$ environ, on peut évaluer le stock total de minéraux essentiels à : de $\mathrm{Ca}: 269 \mathrm{Kg} ; \mathrm{Mg}: 101 \mathrm{Kg}$; de K : $1058 \mathrm{Kg} ; \mathrm{P}: 134$ $\mathrm{Kg}$ et $\mathrm{Fe}: 63 \mathrm{Kg}$.

De nombreux travaux portent sur l'étude de la décomposition des feuilles de ligneux du bord des eaux ou des macrophytes. Moins nombreux sont les travaux sur la décomposition d'espèces colonisatrices des zones momentanément exondées, comme sur les rivages fluviaux ou les zones de marnages des lacs et réservoirs.

Tableau 6. Suivi de mise en solution des éléments participant à la balance ionique lors de la décomposition de Rorippa en cristallisoir. (Pour les anions et cations, valeurs en ppm. Les dosages ont été réalisés en chromatographie ionique type DIONEX).

Table 6. Release of essential elements of ion balance during an experimental decomposition of Rorippa in water.

\begin{tabular}{|c|c|c|c|c|c|c|c|c|c|c|c|c|c|}
\hline & & pH & $\begin{array}{l}\text { Cond. } \\
\mu \mathrm{S} / \mathrm{cm}\end{array}$ & $\mathbf{F}^{-}$ & $\mathrm{Cl}^{-}$ & $\mathrm{NO}_{2}^{-}$ & $\mathrm{NO}_{3}{ }^{-}$ & $\mathrm{HPO}_{4}{ }_{4}{ }^{2 \cdot}$ & $\mathrm{SO}_{2}{ }^{2}$ & $\mathrm{NH}_{4}{ }^{+}$ & $\mathbf{K}^{+}$ & $\mathrm{Ca}^{2+}$ & $\mathbf{M g}^{2+}$ \\
\hline 7.7 .95 & $\mathrm{~J} 1$ & & & & & & & & & & & & \\
\hline $\begin{array}{l}17 \mathrm{~h} 40 \\
\text { Eau Der }\end{array}$ & & 5,70 & 0030 & 0 & 0,44 & - & - & - & 0,04 & 0,10 & 0,01 & - & - \\
\hline 7.7 .95 & J1 & 6,26 & 0070 & 0,37 & 1,05 & 0,07 & 0.13 & - & $.0,80$ & 0,18 & 0,53 & - & 0,10 \\
\hline $17 \mathrm{~h} 40^{\prime} 3$ & & & & & & & & & & & & & \\
\hline $\begin{array}{l}7.7 .95 \\
18 \mathrm{~h}\end{array}$ & $\mathrm{~J} 1$ & 7,80 & 0880 & 0,30 & 1,08 & - & 0,03 & - & 6,09 & 1,73 & 6,80 & 0,90 & 1,60 \\
\hline $\begin{array}{l}10.7 .95 \\
13 \mathrm{~h}\end{array}$ & $\mathbf{J 3}$ & 8,01 & 1120 & 0,30 & 2,25 & 0,05 & 0,05 & 0,19 & 14,99 & 5,78 & 8,40 & 2,10 & 4,34 \\
\hline $\begin{array}{l}11.7 .95 \\
17 \mathrm{~h} 30\end{array}$ & J4 & 8,15 & 1420 & 0,42 & 8,04 & - & 0,12 & 0,42 & 24,74 & 4,30 & 10,25 & 2,60 & 5,50 \\
\hline $\begin{array}{l}12.7 .95 \\
12 \mathrm{~h} \\
\end{array}$ & $\mathrm{~J} 5$ & 8,23 & 2350 & - & 3,10 & - & 0,03 & 1,22 & 29,92 & 13,5 & 11,40 & 3,20 & 6,70 \\
\hline
\end{tabular}


Tableau 7. Liste récapitulative des espèces citées dans l'article. La nomenclature est celle de Bolos et al. (1990).

Table 7. List of plant species recorded. Nomenclature from Bolos et al. (1990).

Achillea ptarmica L. subsp. pyrenaica (Sibth.) Rouy Alopecurus geniculatus L. subsp. fulvus (Sm.) Trab: Anthyllis gr. vulneraria L.

Arabidopsis thaliana (L.) Heynh.

Armeria alliacea (Cav.) Hoffms. \& Link

Artemisia absinthium L.

Artemisia vulgaris L.

Betula pubescens Ehrh.

Briza media L.

Capsella bursa-pastoris L.

Carex sp. pl.

Catabrosa aquatica (L.) Beauv.

Chamaespartium sagittale (L.) P. Gibbs

Conyza cf. canadensis (L.) Cronq.

Deschampsia caespitosa (L.) Beauv.

Descurainia sophia (L.) Webb ex Prantl

Epilobium cf. hirsutum L.

Equisetum sp.(pl. ?)

Filipendula ulmaria (L.) Maxim.

Galeopsis sp:

Galium cf. pumilum Murray

Galium verum L. subsp. verum

Genista anglica L.

Glyceria fluitans (L.) R. Br.

Gnaphalium uliginosum L.

Holcus mollis $\mathrm{L}$.

Juncus bufonius L.

Juncus cf.conglomeratus L.

ou plus probablement cf. effusus L.

Juncus sp.

Lamium amplexicaule $\mathrm{L}$.

Leontodon autumnalis $\mathrm{L}$.

Lotus sp.

Lychnis flos-cuculi $\mathrm{L}$.

Matricaria maritima L. subsp. inodora (L.) Dostal

Mentha longifolia (L.) Huds.
Orchis majalis Reichenb.

Paronychia polygonifolia (Vill.) DC.

Pins hybrides X Pinus sylvestris L. $\mathrm{x}$ P. mugo

Turra subsp. uncinata (Ramond ex DC.) Domin.

Poa annua L.

Poa compressa $\mathbf{L}$.

Poa pratensis $\mathrm{L}$.

Poa trivialis $\mathbf{L}$.

Polygonum aviculare $\mathrm{L}$.

Polygonum persicaria L.

Populus nigra L.

Prunella vulgaris $\mathrm{L}$.

Ranunculus (subgen. Batrachium ) sp.

Ranunculus repens $\mathrm{L}$.

Rorippa islandica (Gunnerus) Borbas

Rumex sp. (subgen. Rumex)

Sagina apetala And. subsp. erecta (Homem.)

Hermann

Salix sp.

Salix pentendra L.

Scleranthus sp.

Senecio vulgaris $\mathrm{L}$.

Solanum lycopersicum L.

Sonchus sp:

Spergula sp.

Spergularia rubra (L.) J. \& C. Presl. subsp.

nubra

Stellaria alsine Grimm

Tanacetum vulgare $\mathrm{L}$.

Thlaspiarvense $\mathrm{L}$. subsp. arvense

Trifolium sp. pl.

Typha sp.

Urtica dioica $\mathrm{L}$.

Veronica beccabunga L.
Devant la multiplication des vidanges de réservoirs, il nous semble important d'essayer de préciser l'impact de la biomasse végétale ennoyée sur la «qualité» de l'eau, après remplissage du réservoir. Ceci rejoint les problèmes abordés lors des études des zones d'interface terre-eaù (Wetzel 1990).

Pour cette étude du réservoir de Puyvalador, lancée dans l'urgence parce que la colonisation végétale, spectaculaire, était déjà en cours, il n'a pas été possible d'appliquer une méthodologie suffisamment rigoureuse.

Cette première approche montre cependant que certains traceurs pourraient aider au suivi du devenir de la végétation ennoyée lors de la mise ou remise en eau d'un barrage, notamment le potassium et le phosphore mais d'autres traceurs comme le COD, les sucres, l'oxygène dissous et l'azote pourraient également être pris en compte. 
Les mécanismes de relargage sont certainement plus complexes car il faut aussi tenir compte de la libération de ces éléments par le sédiment exondé, séché, puis réhumidifié lors du remplissage du réservoir. Un exemple de cette complexité a été donné sur ce même réservoir de Puyvalador, pour ce qui concerne la solubilisation du phosphore lors des variations importantes du niveau d'eau (Fabre 1988a).

A l'issu de cette étude, on peut envisager un processus méthodologique plus rigoureux d'un futur suivi de vidange de réservoir en trois phases :

1) Avant la vidange : une étude détaillée du site, tant sur le plan géographique que géomorphologique et géologique, sur documents cartographiques et photographiques ; suivi des baisses successives du plan d'eau avant la vidange totale accompagné d'observations sur le terrain, de relevés divers et de photographies à différentes échelles.

2) Après la vidange, un premier état des lieux avec prélèvements des sédiments; une première photographie aérienne du site pour enregistrer l'état initial.

3) Les mois suivant la remise en eau, mesures de quelques paramètres essentiels (teneurs en $\mathrm{P}, \mathrm{K}, \mathrm{N}, \mathrm{O}_{2}$, $\mathrm{COD}$, sucre) pour préciser l'éventuel impact de la minéralomasse végétale lors de la reconstitution des équilibres biologique et physico-chimique au sein de la nouvelle masse d'eau.

Notons également que, jusqu'à la remise en eau, deux observations mensuelles sont souhaitables pour suịvre l'évolution du milieu.

Quelques jours avant la remise en eau, une photographie aérienne verticale est indispensable pour une cartographie précise des surfaces enherbées. A la même période, des échantillons de végétation bien choisis devront permettre un bilan fiable de la biomasse des principaux groupements végétaux. Sur ces échantillons, la minéralomasse sera déterminée. Le stock des principaux éléments minéraux participant au cycle biogéochimique pourra ainsi être estimé avec une approximation raisonnable.

Parallèlement, deux autres expériences devraient être envisagées in situ lors de la remise en eau :

le suivi de la minéralomasse restante d'un stock de matériel végétal bien choisi et immergé dans des filets spéciaux, selon un protocole adapté de récupération;

le suivi des concentrations dans l'eau de la retenue de quelques éléments essentiels au cours du remplissage.

Cette première approche pour une meilleure compréhension du cycle biogéochimique de certains miné- raux essentiels devra être complétée, dans le futur, en vue d'une éventuelle prise en compte de ces processus dans un modèle de fonctionnement d'un plan d'eau après vidange.

\section{Remerciements}

Les auteurs tiennent à remercier :

Mme P. Gouaux (CESBIO, Toulouse III) qui mis à leur service ses compétences en traitement d'images et n'oublient pas également l'aide que leur ont apporté Mr. J.J. Troubat (ORSTOM) et Mme M. Aizpuru (LET, Toulouse III) lors de la réalisation des deux cartes couleur :

MM. S. Payré et A. Guillot, responsables EDF de la Centrale de Nantillat qui ont aimablement mis à leur disposition les photos couleurs réalisées par IGN quelques jours après la vidange ;

Mr. J.C. Ferrer, Directeur du GEH d'Axat qui nous a aimablement autorisé à publier les données EDF concernant les cotes de la retenue de Puyvalador ;

Mme D. Galy qui a assuré la saisie du manuscrit.

\section{Travaux cités}

Allenby K. G. 1981, - Some analyses of aquatic plants and their waters. Hydrobiologia, 77 : 177-189.

Baudière A, \& Serve L. 1976. - Les groupements à Ligularia sibirica Cass. du Capcir (haute vallếe de l'Aude, Pyrénées-Orientales). Bull. Soc. Bot. Fr, $123: 167-174$.

Bolos (de) O., Vigo J., Masalles R., \& Ninot J. 1990. - Flora manual dels paisos catalans, Barcelona: $1247 \mathrm{p}$.

Bourraqui-Sarre L. 1997. - Contribution à l'étude de la flore accompagnatrice des moissons et colonisatrice des jachères de la Cerdagne française et du Capcir (Pyrénées orientales). Le Monde des Plantes, 458, 1er trim. 1997, 24-27.

Butcher D.P., Labadz J.C., Potler A.W.R. \& White P. 1993. - Reservoir sedimentation rates in the southern Pennine region, Uk. Geomorphology and sedimentology of Lakes and Reservoirs. J. Mcmanus and R.W. Duck (eds). J. Wiley \& Sons Ltd New-York : 73-92.

Chassagne J.M. 1994. — Demande d'autorisation pour la vidange decennale du barrage de Puyvalador. Rapport EDF, 73p. + annexes.

Chauvet E. 1989. - Production, flux et décomposition des litières en milieu alluvial. Dynamique et rôle des hyphomycètes aquatiques dans le processus de décomposition. Thèse Doctorat, Université Toulouse III, $239 \mathrm{p}$.

Dagnac J. 1994. - Bilan des dépôts sédimentaires dans le réservoir de Pareloup (Aveyron, France) Hydroécol. Appl. t. 6, vol. 1-2, pp. 59-85.

David C. 1994. - Etudes sur les sédiments de deux lacs de barrage aveyronnais : Bage et Pont-de-Salars. Diplôme d'Etudes Approfondies, Université Toulouse III : $47 \mathrm{p}$.

Fabre A. 1988a. - Hétérogénéité des sédiments dans un réservoir à fort marnage et étude expérimentale de la solublisation du phosphore. Thèse Doctorat, Université Toulouse 111, 192 p.

Fabre A. 1988b. - Exprimental studies on some factors influencing phosphorus solubilisation in connexion with the drawdown of a reservoir. Hydrobiologia, 159 : 153-158.

Fraissé Th., \& Maman L. 1993. - Végétalisation des zones de marnage. Revue de l'Agence de l'Eau Adour-Garonne, ${ }^{\circ} 56$ : 19-22.

Gaussen H. 1972. - Carte de la végétation de la France. Perpignan ( $2^{\text {ème } e ́ d .) . ~ C a r t e ~ a u ~} 1 / 200000$ avec notice sommaire. Service de la Carte de la Végétation, Toulouse (C.N.R.S.). 
Gerloff G. C. \& Fishbeck K. A. 1969. - Quantitative cation requirements of several green and blue-green algae. J. Phycol., $5: 109$ 114.

Gerloff G. C. \& Krombholz P. H. 1996. - Tissue analysis as a measure of nutrient availability for the growth of angiosperm aquatic plants. Limnol. Oceanogr., 11(4) : 529-537.

Helmer de Almeida C. 1994. - Importance de la dénitrification et des relations aux sédiments dans le fonctionnement des lacs de gravière. Thèse Doctorat, Université Toulouse III : 299 p.

Hutchinson G. E. 1975. - The Chemical Ecology of Freshwater Macrophyts. In Treatise of Limnology. Vol. III. Limnological Botany. J. Wiley and Sons, New-York : 660p.

Kem-Hansen U. \& Dawson F. H., 1978. - The standing crop of aquatic plants of lowland streams in Denmark and the inter-relationships of nutrients in plant, sediment and water. Pro $c$. EWRS 5 th Symp on Aqua tic Weeds : 143-150.

Kormondy B. J., 1976. - Concepts of Ecology. Prencetice-Haîl, mc., Englewood Cliffs, New Jersey, 238 p.

Labroue L., Capblancq J., Salençon M.J., Toureng J.N. \& Mur C. 1994. - Evolution saisonnière des éléments minéraux nutritifs $(\mathrm{P}, \mathrm{N}, \mathrm{Si})$ et de l'oxygène dissous dans le lac de Pareloup. $H y-$ droécol. Appl. 6 (1-2) : 87-114.

Langlade L. R. 1996. - Colonisation de la végétation pionnière des bancs de galets en rivière. Revue de l'Agence de l'Eau Adour-Garonne, $\mathrm{N}^{\circ} 67,19-22$.

Leuven R.S.B.W. \& Wolfs W.J. 1988. - Effects of water acidification on the decomposition of Juncus bulbosus L. Aquatic Botany, 31: 57-81.

Mackereth F.J.H., 1957. - Chemical analysis in ecology illustrated from lake district tarns and lakes. 1. Chemical analysis. Proc. Linn. Soc. Lond., 167(2) : 159-164.

Mas P. 1959. - Exploitation des étangs de la Dombes. Dipl. Ecole Nat. Agro. Montpellie :, 140p.

Melillo J.M., Naiman R. J., Aber J. D \& Eshleman K.N. 1983. The influence of substrat quality and stream size on wood decomposition dynamics. Oecologia, $58: 281-285$.
Meybeck M. 1976. — Dissolved and suspended matter carried by tirers, time and space variations, and world balance. Interactions between sediments and freshwater, Junk \& Pudoc, Amsterdam : 25-32.

Pattee E., Bomard C., \& Mourelatos S. 1985. - La décomposition des feuilles mortes dans le réseau fluvial du Rhône : influence du milieu et principaux agents responsables.Sci. Eau, 5 : 45-74.

Petitjean F. 1981. - Etude de l'herbier à Ranunculus fluitans d'Argentat sur Dordogne.Thèse $3^{\mathrm{eme}}$ Cycle, U.P.S. Toulouse II : $130 \mathrm{p}$.

Quennerstedt N. 1958. -- Effect of water level fluctuation on lake vegetation. Verh. Internat. Verein. Limnol., XIII : 901-906.

Rey P., Dupias G. \& Izard M. 1973. - Excursion dans les Pyrénées de l'Est (Vallée de l'Aude, Donezan, Capcir, Cerdagne). Service de la Carte de la Végétation, Toulouse (CNRS), 8 p. multigraph.

Short F.Y. 1987. - Effects of sediments nutrients on sea grasses : litterature review and mesocosm experiment. Aquatic Botany, 27 ; 41-57.

Smith P. F. 1962. - Mineral analysis of plant tissues. Ann. Rev. Plant Physiol., 13 : 81-108.

Suberkropp K., Godshalk G. L. \& Klug M.J. 1976. - Changes in the chimical composition of leaves during processing in a woodland stream. Ecology, $57: 720-727$.

Terrisse A. \& Terrisse J. 1976. - Compte-rendu de la troisième session extraordinaire de la S.B.C.O. tenue à Mijanès (Ariège) du 10 au 15 juillet 1976. Bull. Soc. bot. Centre-Ouest, n. sér. 7: 51-70.

Terrisse A. 1996. - La végétation d'un lac de barrage provisoirement asséché (Etang de Puyvalador, Pyrénées-Orientales). Bull. Soc. bot. Centre-Ouest, n. sér. 27 : 59-62.

Viers G. 1968. - Carte du relief glaciaire des Pyrénées. Mont-Louis Est., Institut de Géographie Daniel Faucher Toulouse, Rev. Géog. Pyr. Sud-Ouest. Supplément au t.XXXIX.

Wetzel R. G. 1990. - Land-water interfaces : Metabolic and limnological regulators. Verh. Internat. Verein. Limnol., 24 : 25-37. 